\title{
Deterioration of Baroreceptor Reflex by Transient Global Cerebral Ischemia in Dogs
}

\author{
Junichi KURIHARA, Hiroyuki NISHIMURA, Nobuyuki ODA \\ and Hitoshi KATO
}

Department of Pharmacology. Faculty of Pharmaceutical Sciences, Teikyo University, Sagamiko, Kanagawa 199-01, Japan

Accepted October 28, 1988

\begin{abstract}
Influence of transient global cerebral ischemia on baroreceptor reflex sensitivity (BRS) was investigated in anesthetized dogs. Cerebral ischemia was produced by the combined occlusions of the left subclavian (LSA) and the brachiocephalic (BCA) arteries with preceding ligations of the intercostal arteries (ICA). BRS was assessed by phenylephrine-induced reflex bradycardia. Ischemia of 5and 10-min duration produced a significant decrease in BRS during the reperfusion period of 60-120 min. On the other hand, such a change was not observed following the ischemia of less than 2-min or occlusions of LSA and BCA without preceding ligations of ICA. Heart rate response to the electrical stimulation of the vagal afferent nerve was attenuated by 10 -min ischemia, while response to the vagal efferent nerve stimulation and ECG parameters were not influenced. These results indicate that some regions vulnerable to the relatively short duration of cerebral ischemia may be involved in the central pathway of the baroreflex mechanism.
\end{abstract}

Since the central nervous system plays an important role in the regulation of the cardiovascular system, it is proposed that its dysfunction may be responsible for cardiovascular disorders such as abnormalities in the electrocardiogram (ECG) (1-3), arrhythmias (3) or deterioration of circulatory reflexes (4-6). which may critically influence the clinical outcome in patients with cerebrovascular diseases. In a large number of clinical studies, however, a direct cause-effect relationship between the cerebrovascular diseases and the cardiovascular disorders has not always been clear due to the diversities of the nature of the patients, type of disease, site of lesion, the influences of the concurrent diseases or undergoing therapy as well as difficulty in finding matched control subjects. Therefore, systematic investigation with an appropriate animal model may be useful for a more precise understanding of the influence of the cerebrovascular diseases on the regulation of the cardiovascular system. In this context, the present study was carried out to examine the influence of transient global cerebral ischemia on baroreceptor-mediated control of heart rate, which is one of the typical circulatory reflexes via the central nervous system.

\section{Materials and Methods}

Mongrel dogs of either sex weighing about 10 to $20 \mathrm{~kg}$ were anesthetized with pentobarbital sodium, $32 \mathrm{mg} / \mathrm{kg}$, i.v., and a supplemental dose of $3.2 \mathrm{mg} / \mathrm{kg} / \mathrm{hr}$, i.v. Animals were artificially ventilated with room air in a tidal volume of $20 \mathrm{ml} / \mathrm{kg}$ at a rate of 25 breaths/min. Since the ischemic insult may produce an intense movement of the trunk due to excitation of the respiratory center, which may disturb the stable recordings of the parameters, animals were immobilized with suxamethonium chloride, $2 \mathrm{mg} / \mathrm{kg}$, i.v., and 1 $\mathrm{mg} / \mathrm{kg} / \mathrm{hr}$, i.v., supplementally. The $P_{\mathrm{O}_{2}}, P_{\mathrm{co}_{2}}$ and $\mathrm{pH}$ of blood samples drawn from the right saphenous artery were measured by a blood gas analyzer (Instrumentation Laboratory, 213 and 326, Lexington, U.S.A.), and appropriate volume of $\mathrm{O}_{2}$ and $\mathrm{CO}_{2}$ gasses were provided via a tracheal tube to maintain $\mathrm{P}_{\mathrm{O}_{2}}$ and $\mathrm{P}_{\mathrm{co}_{2}}$ at about 100 and $35 \mathrm{mmHg}$, 
respectively.

Arterial blood pressure was measured from the left femoral artery by means of a pressure transducer (Nihon Kohden, TP-200T, Japan). Heart rate was measured by a heart rate counter (Nihon Kohden, AT-600G) triggered by the arterial blood pressure wave or lead II ECG. A precalibrated flow probe was placed around the right vertebral artery at the C-7 level, and blood flow was measured by an electromagnetic flowmeter (Nihon Kohden, MFV-2100). An indwelling catheter was inserted into the cisterna magna through the foramen magnum and intracisternal pressure was measured by means of a pressure transducer (Nihon Kohden, TP-200T). Rectal temperature was maintained at about $38^{\circ} \mathrm{C}$ using a heating pad and lamp.

Production of cerebral ischemia: Thoracotomy was performed at the left fifth intercostal space. Approximately 12 intercostal arteries descending along the thoracic aorta were ligated to obstruct the collateral blood flow to the brain. Then the head was rigidly secured by means of a stereotaxic apparatus in a sphinx-like position. Craniotomy was performed, and a platinum electrode was inserted into the marginal gyrus, $1 \mathrm{~cm}$ frontal to the external meatus, to a depth of 1 to $2 \mathrm{~mm}$. The skull was sealed with dental cement. A plate type silver-silver chloride electrode was placed under the skin of the neck as a reference electrode. Regional cerebral cortex blood flow ( $\mathrm{CCBF}$ ) was measured by the hydrogen clearance method $(7,8)$ to confirm the reduction of cerebral blood flow by the ischemic procedure. The electroencephalogram (EEG) was recorded by means of stainless steel electrodes screwed bilaterally into the skull $1 \mathrm{~cm}$ frontal to the hydrogen electrode. In some animals, EEG frequency analysis was carried out using a frequency analyzer (Nihon Kohden, OEE-7102).

Global cerebral ischemia was produced by the occlusion with clamps of the left subclavian artery and the brachiocephalic artery as close as possible to the aortic arch. According to the pooled data in our laboratory, this procedure produced EEG flattening in 56 out of 80 animals. In these animals, no detectable amount of hydrogen gas appeared in the cerebral cortex during the ischemic insult, indicating that residual blood flow was extremely low at least in the region where rCBF was measured. In the rest of animals, EEG amplitude was only reduced without flattening or little affected, and a considerable amount of hydrogen gas appeared in the cerebral cortex during the ischemic procedure. These animals whose EEG persisted during the ischemic procedure were eliminated from the present study.

The first series of experiments consisted of 14 animals equally divided into 2 groups. One group received $10-\mathrm{min}$ ischemic insult, and the other served as the incomplete occlusion group, in which the brachiocephalic and the left subclavian arteries were occluded for $10 \mathrm{~min}$ without preceding ligation of the intercostal arteries. In the next series of experiments, another 21 animals were equally divided into 3 groups and assigned to different duration of ischemic insult, i.e., 0. 2 and $5 \mathrm{~min}$; the 0 -min ischemia group was denoted as the sham-operated group.

Measurement of baroreceptor reflex sensitivity (BRS): Baroreceptor reflex was assessed by the bolus injections of 3 to 5 doses of phenylephrine within the dose range of 0.3 to $10 \mu \mathrm{g} / \mathrm{kg}$, i.v. Phenylephrine-induced increase in pulse interval (msec) was correlated with the increase in mean arterial blood pressure $(\mathrm{mmHg})$ by the method of least squares. Since no parallel shift of the linear regression line was observed in the present study, the slope of the regression line (msec/ $\mathrm{mmHg}$ ) was utilized as a measure of BRS. BRS was measured before ischemia and during the period of 60 to 90,120 to 150 , and 180 to $210 \mathrm{~min}$ reperfusion.

Electrical stimulation of the vagal afferent and efferent nerves: An additional 10 animals were used in this separate experiment; 5 animals were used for the vagal afferent nerve stimulation and the rest for the efferent nerve stimulation. The left vagal nerve in the cervical region was cut and either the central or peripheral end was electrically stimulated using a square wave stimulator (Nihon Kohden, SEN-7103). Electrical stimuli of $0.3 \mathrm{msec}$ duration, 1 to $10 \mathrm{~Hz}, 5$ to $8 \mathrm{~V}$ were applied to the afferent nerve for $10 \mathrm{sec}$; and those of $0.3 \mathrm{msec}$ duration, 1 to $10 \mathrm{~Hz}, 2$ to 5 $\checkmark$ were applied to the efferent nerve for $5 \mathrm{sec}$. 
All animals received $10-\mathrm{min}$ ischemic insult, and stimuli were applied before ischemia and during the period of 90 to 120,150 to 180 , and 210 to $240 \mathrm{~min}$ reperfusion. Additionally. BRS was also measured as mentioned above.

Statistical analyses: Values in different groups were analyzed by the one-way analysis of variance and the wholly-significantdifference method, unless otherwise indicated in the text. Differences between the mean values before and after ischemia were analyzed by the paired Student's $t$-test. Differences in the slope of the regression lines in Figs. 3 and 4 were analyzed by the analysis of covariance. Differences giving $P<0.05$ were regarded as statistically significant.

\section{Results}

Comparison between 10-min cerebral ischemia and incomplete occlusion: Ischemia by clamping the brachiocephalic and the left subclavian arteries produced abrupt increases in diastolic and systolic blood pressure and pulse pressure, as shown in Fig. 1. In most cases of the ischemia group, the initial in- crease in heart rate by ischemia was followed by bradycardia or some kind of arrhythmia. Therefore, the pattern of change in heart rate was highly variable among the animals. These abnormal states of blood pressure and heart rate were normalized by the removal of clamps, i.e., by reperfusion. On the other hand, as shown in Fig. 2, the extent of increase in blood pressure in the incomplete occlusion group was significantly $(P<0.001$ by unpaired Student's $t$-test) smaller than that in the ischemia group; maximum responses were $64 \pm 9$ vs. $128 \pm 8 \mathrm{mmHg}$. Furthermore, heart rate persistently increased without showing any arrhythmias during the incomplete occlusion period.

Intracisternal pressure and vertebral blood flow were transiently increased by the reperfusion; these changes may reflect the reactive hyperperfusion state in the brain. The extent and duration of increase in intracranial pressure in the ischemia group were very markedly greater than those in the incomplete occlusion group. However, no significant change in intracisternal pressure

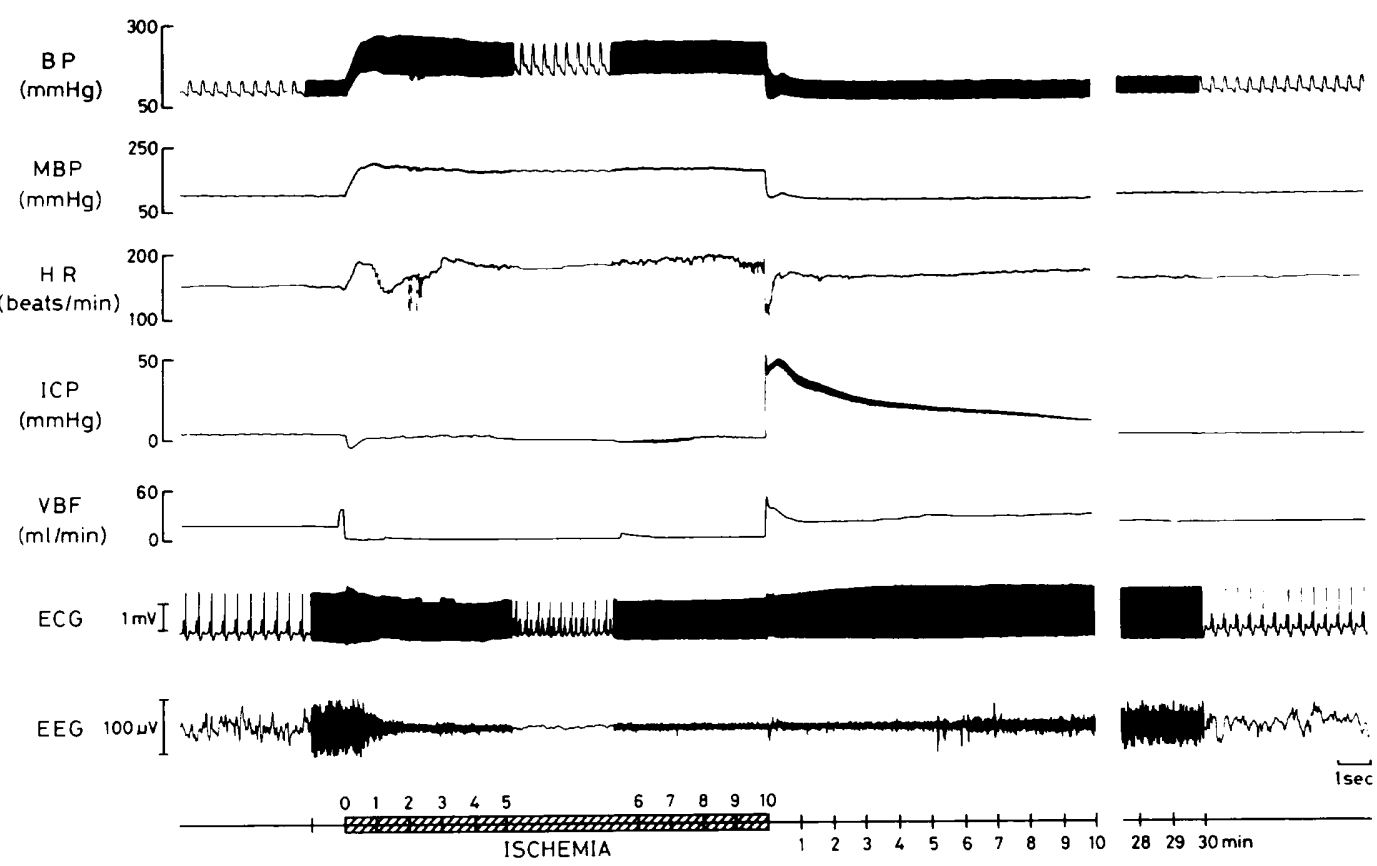

Fig. 1. The influence of 10-min global cerebral ischemia on arterial blood pressure (BP), mean arterial blood pressure (MBP), heart rate (HR), intracisternal pressure (ICP), right vertebral blood flow (VBF). electrocardiogram (ECG) and electroencephalogram (EEG) in anesthetized dog. 

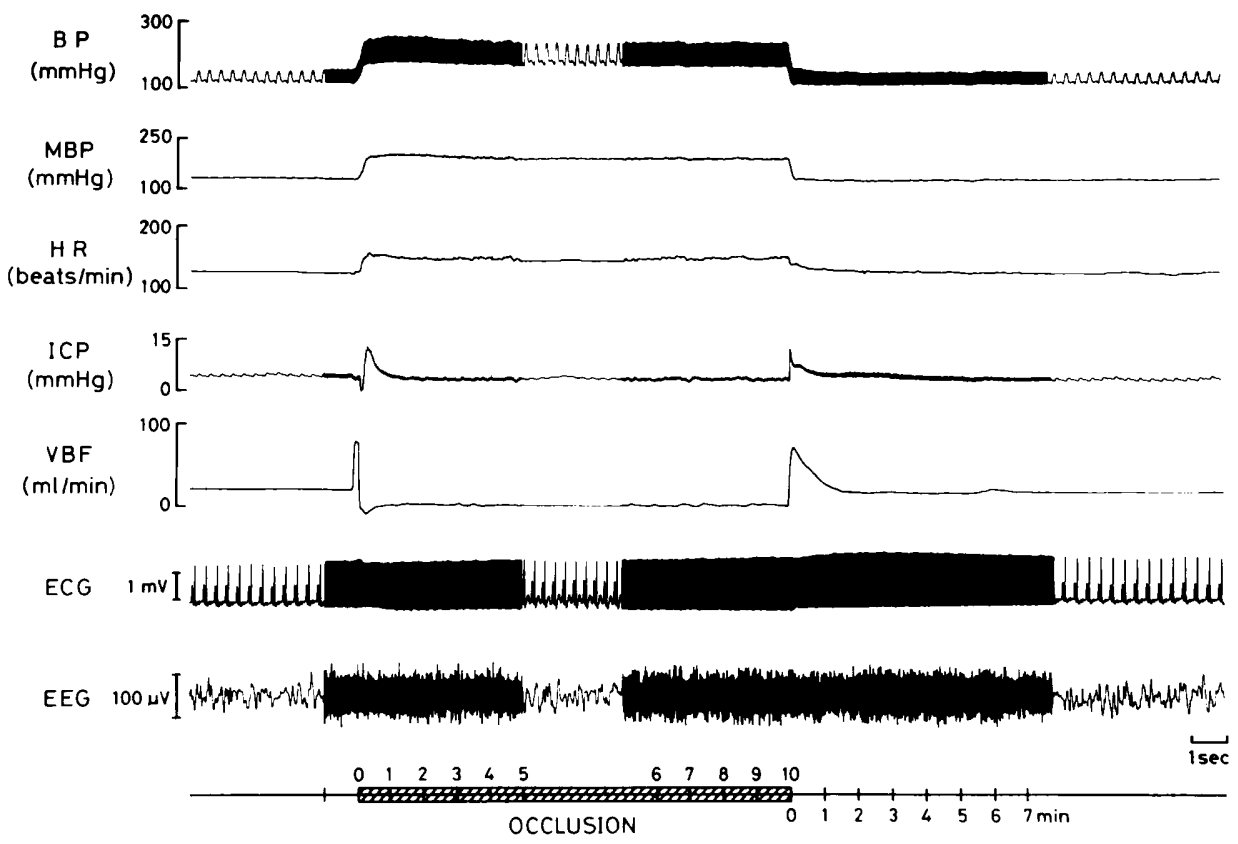

Fig. 2. The influence of 10 -min incomplete occlusion. Abbreviations are as in Fig. 1.

was observed during the reperfusion period of 60 to $180 \mathrm{~min}$.

EEG in the ischemia group flattened within $1 \mathrm{~min}$ of ischemic insult, while no noticeable change in EEG was observed during the incomplete occlusion period. EEG started to recover a few minutes after the reperfusion. However, the recovery of EEG in the 10-min ischemia group seemed to be incomplete; the amplitude of the EEG tended to be smaller than that before ischemia.

Figures 3 and 4 summarize the phenylephrine-induced reflex bradycardia in the 10min ischemia and 10-min incomplete occlusion groups, respectively. There was no difference between BRS before ischemia and that before incomplete occlusion. BRS during the reperfusion period following 10-min ischemia was statistically significantly smaller than that before ischemia, and this attenuation persisted throughout the experimental period, while no decrease in BRS was observed in the incomplete occlusion group.

Influence of different duration of cerebral ischemia: Influence of cerebral ischemia of 0,2 and 5 min was compared with that of 10 $\mathrm{min}$ ischemia to investigate the dependency of post-ischemic decrease in BRS on the duration of ischemia.

BRS in the 2-, 5- and 10-min ischemia and sham-operated groups before ischemia were $2.16 \pm 0.28,1.92 \pm 0.24,2.14 \pm 0.17$ and $1.90 \pm$ $0.33 \mathrm{msec} / \mathrm{mmHg}$ (mean \pm S.E.M., $n=7$ for each group), respectively, and there was no significant difference among them. As shown in Fig. 5, 5-min ischemia produced a significant decrease in BRS to the same extent as 10-min ischemia, while ischemia of less than 2 min did not affect BRS.

EEG in the 2- and 5-min ischemia groups recovered by reperfusion earlier than that in the 10-min ischemia group. In most animals, EEG started to recover just after the reperfusion and completely recovered within several minutes; EEG recovery speed after 2min ischemia was clearly higher than that after 5-min ischemia. Additionally, as shown in Table 1. EEG frequency analysis indicated no significant change in the cortical EEG power spectrum during 5 to $180 \mathrm{~min}$ of reperfusion following 5 -min ischemia, although the delta component just after the onset of reperfusion (0 to $2 \mathrm{~min}$ ) was significantly decreased. Thus, the post-ischemic attenuation of BRS in the 5-min ischemia group was found to be accompanied by no significant 

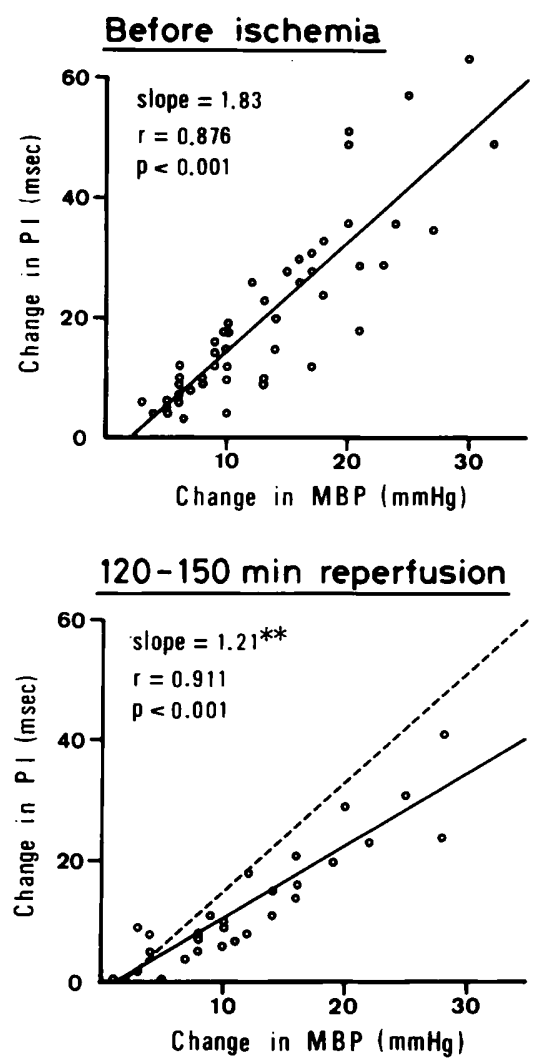
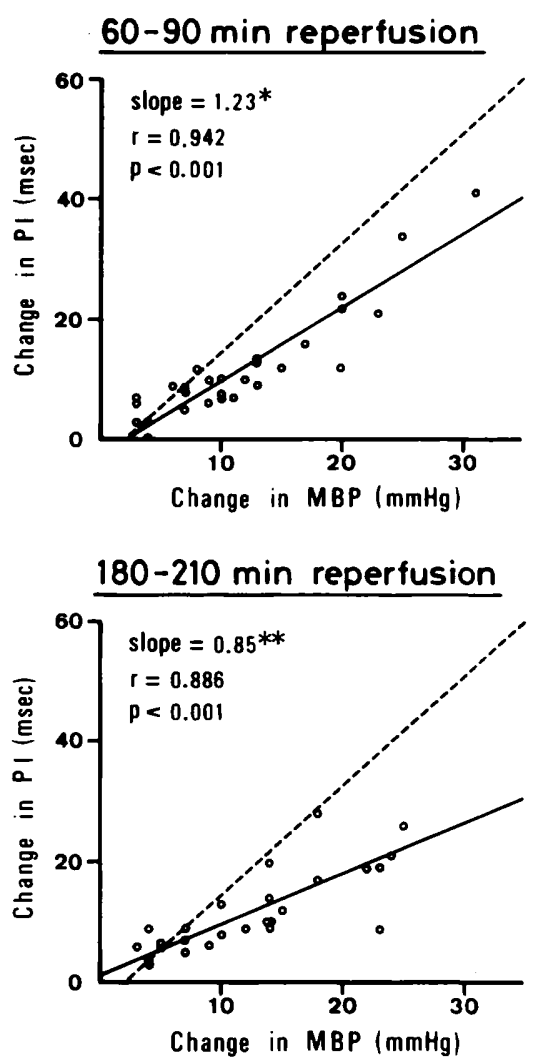

Fig. 3. Influence of 10-min cerebral ischemia on phenylephrine-induced reflex bradycardia. Abscissa: change in mean arterial blood pressure (MBP). Ordinate: change in pulse interval (PI). Data from 7 animals are plotted, and the calculated regression lines are indicated as solid lines. Slope, $r$ and $P$ denote the slope, correlation coefficient and rate of risk of linearity of the regression line, respectively. Broken lines are the superimpositions of the regression line before ischemia. ${ }^{*} \mathrm{P}<0.01,{ }^{*} \mathrm{P}<0.005$ : significantly different from the value before ischemia.

change in the cortical EEG.

Influence of cerebral ischemia on heart rate response to the vagal nerve stimulation and ECG: As shown in Fig. 6, electrical stimulation of the vagal afferent or efferent nerve produced frequency-dependent bradycardia; maximum response to the afferent nerve stimulaton was attained at $8 \mathrm{~Hz}$. After 10 -min cerebral ischemia, significant attenuation of response to the afferent vagal nerve stimulation at 6 to $10 \mathrm{~Hz}$ was observed, although the responses to 1 to $4 \mathrm{~Hz}$ stimulation were not affected. This attenuation persisted throughout the experimental period. On the other hand, responses to the efferent vagal nerve stimulation at any of the applied frequencies were not attenuated by $10-\mathrm{min}$ ischemia.

Table 2 summarizes ECG parameters and
BRS in the animals used for the vagal nerve stimulation. Although some kind of arrhymias, manifestation of tall $T$ waves or inversion of $T$ waves were observed during ischemia, neither significant change in ECG wave amplitudes and intervals nor deviation of ST-segment was observed after reperfusion. On the other hand, BRS was significantly decreased by 10-min cerebral ischemia.

\section{Discussion}

The present study confirmed that baroreflex sensitivity during the reperfusion period of 60 to $210 \mathrm{~min}$ following transient global cerebral ischemia of more than 5-min duration was significantly smaller than that before ischemia. Since the decrease in BRS was not observed in the sham-operated or 2-min 


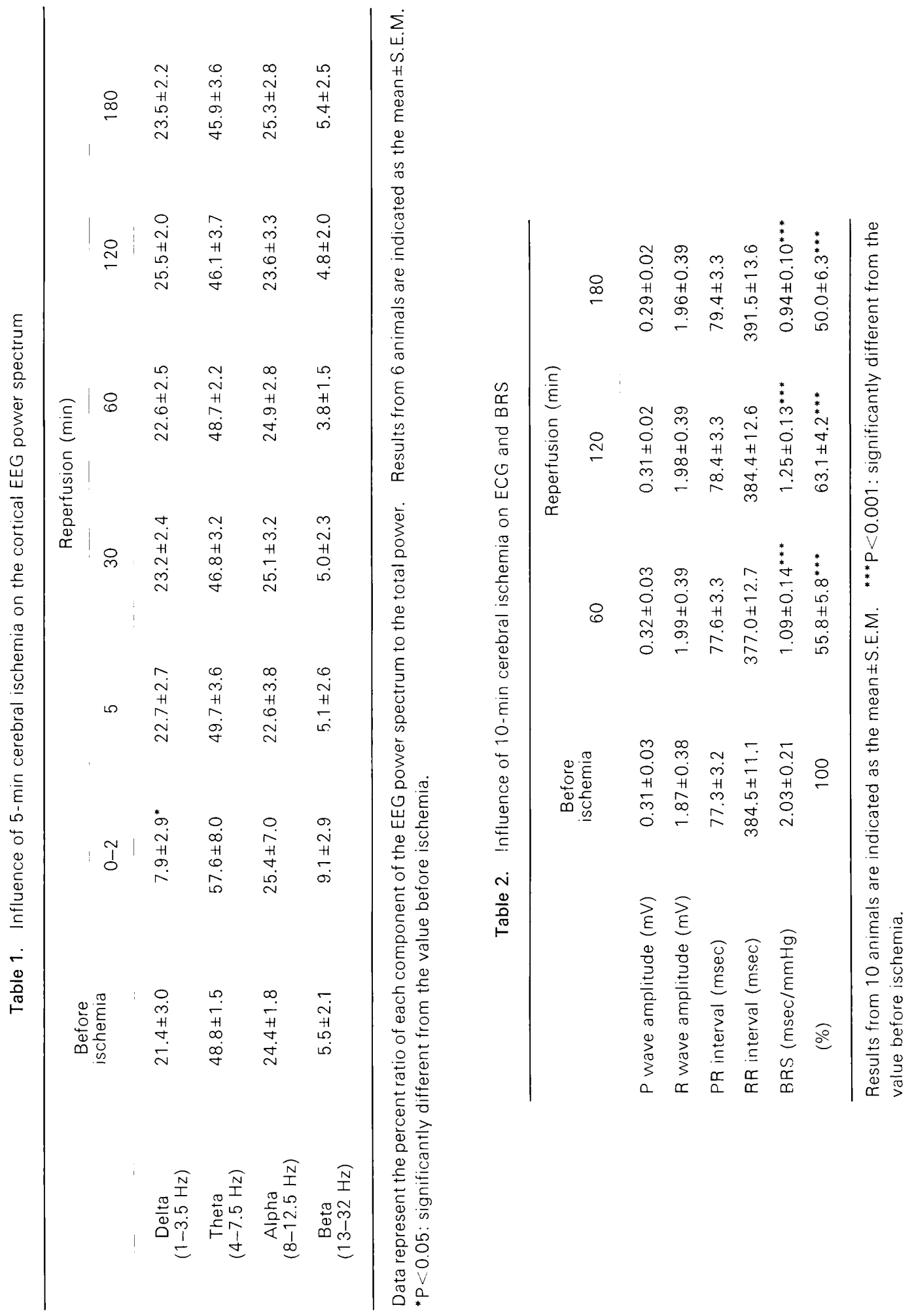


Before occlusion

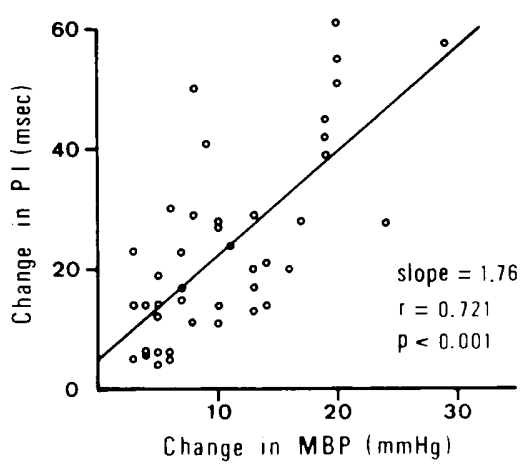

120-150 min repertusion

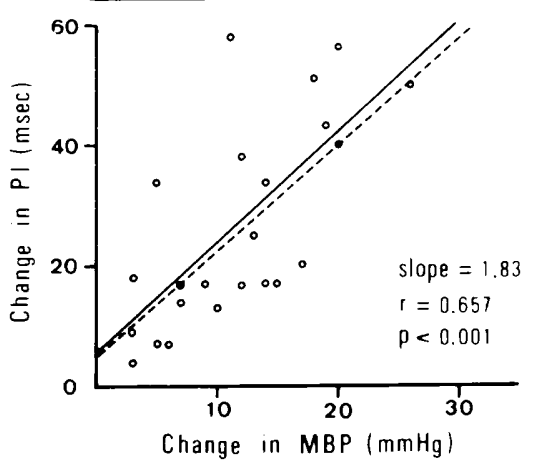

60-90 min reperfusion

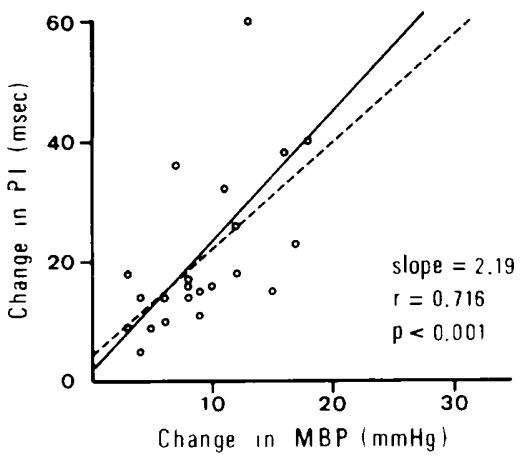

$180-210 \mathrm{~min}$ reperfusion

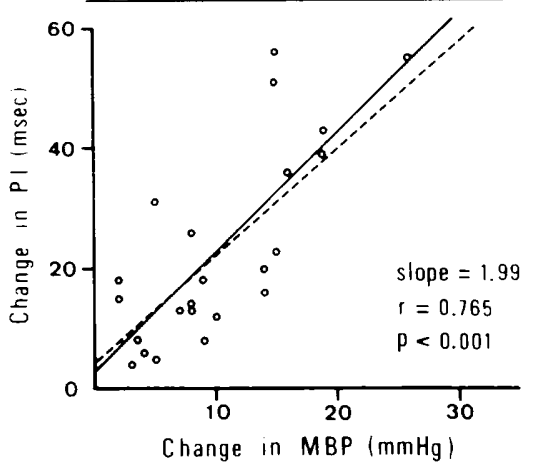

Fig. 4. Phenylephrine-induced reflex bradycardia in the 10-min incomplete occlusion group. Notations are as in Fig. 3.

ischemia group as well as the 10-min incomplete occlusion group, such a change may not be ascribed to any kind of artifact during the ischemic procedure, but rather due to the ischemia itself.

It is suggested that acute stroke may increase sympathetic activity with resultant electrocardiographic abnormalities and myocardial cell necrosis $(3,9)$. Therefore, one might speculate that the observed decrease in BRS might be due to the damage to the cardiac response to the vaga! efferent nerve. However, it was not the case in the present study, because heart rate response to the vagal efferent nerve stimulation was not affected by 10 -min ischemia in spite of the concomitant decrease in the response to the vagal afferent nerve stimulation. Probably, the duration of ischemic activation of the sympathetic system in the present study may be too short to produce such cardiac damages. Thus, the damaged site responsible for the decrease in BRS may be involved in the central pathway of baroreflex. Furthermore, significant attenuation of the responses to the afferent nerve stimulation was observed only at frequencies higher than $6 \mathrm{~Hz}$, which agreed with the finding that the cerebral ischemia decreased the slope of the regression line that correlated increases in blood pressure and pulse interval, with no paraliel shift of the line. These results indicate that the baroreflex mechanism was still capable of counteracting the small change in blood pressure, although the maximum buffering ability had been reduced by the cerebral ischemia.

It has been shown in clinical studies that cerebrovascular disease may be implicated in the deterioration of circulatory reflexes in elderly patients $(4,5)$. However, it seemed to be difficult to conclude which of the two factors, i.e., cerebrovascular disease or aging. 
Before ischemia

120-150 min reperfusion
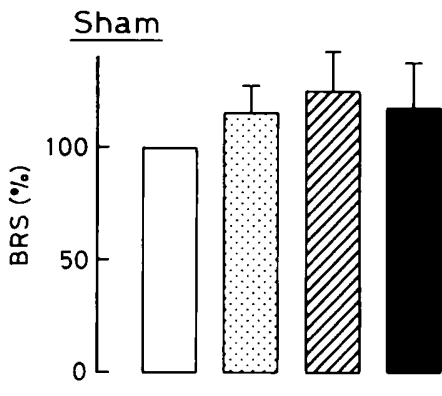

5-min ischemia

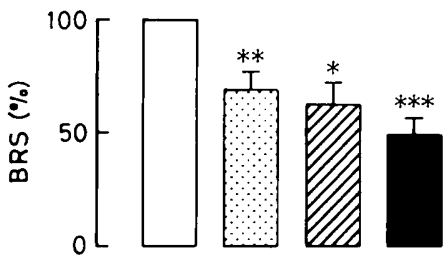

60-90 min reperfusion

180-210 $\mathrm{min}$ reperfusion

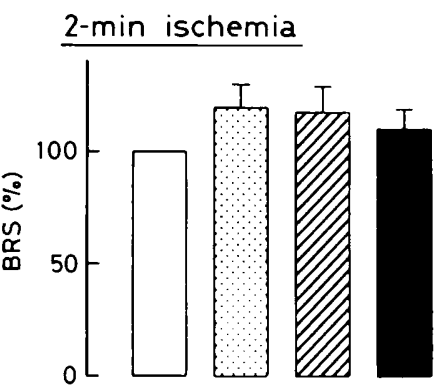

10-min ischemia

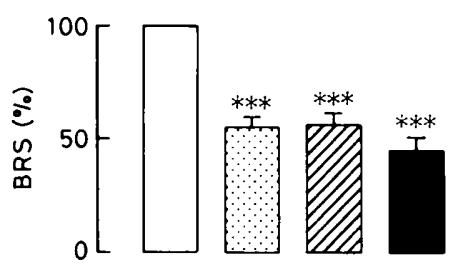

Fig. 5. Influence of cerebral ischemia of different duration on baroreflex sensitivity (BRS). Each group consists of 7 animals. Results are indicated as percentage of the value before ischemia. " $P<0.05$, ${ }^{* * P}<0.01,{ }^{* *} \mathrm{P}<0.001$ : significantly different from the va!ue before ischemia.

was primarily responsible for such impaired circulatory reflexes. In this regard, the present study suggests that cerebrovascular disorder itself may be capable of attenuating the circulatory reflex concerning cardiac performance. This result is consistent with that by Gross (6), who reported that cerebrovascular disease may be responsible for the impaired reflex cardioaccelerator response to Valsalva's maneuver in patients suffering from combined carotid and vertebrobasilar ischemia. Although Gross dealt with only patients with ischemic cerebrovascular disease in the chronic stage. the present result adds the evidence that the attenuation of baroreflex function may occur also in the acute stage following transient global cerebral ischemia. Such an attenuation of circulatory reflex in the acute stage has been observed by Appenzeller and Descarries (4), and they further observed the recovery of baroreflex function concerning reflex vasoconstriction in 4 out of 7 such patients. However, it is not possible from the present shortterm experiment to conclude whether the attenuation of the baroreflex in the present study is also reversible or not.

The major question in the present study is the reason why baroreflex activity was attenuated during the observed reperfusion period following 5 -min ischemia without concomitant impairment of the cortical EEG. First of all, it must be considered that some chronological heterogeneity may exist in the development of neuronal damages by cerebral ischemia, because it has been shown that a certain maturation period of up to several days was necessary for the development of neuronal damages in some selectively vulnerable areas following relatively short duration of ischemia $(10,11)$. In this regard. Suzuki et al. (11) reported that spontaneous neuronal activity of the cortex in gerbils remained within the normal range on the second day after 5 min ischemia, in spite of the concomitant functional death of the CA1 neurons of the hippocampus. On the other hand, however, Smith et al. (12) showed that some morphologica! damages developed in the cerebral cortex during the 7-day recovery period following 4- to $10-\mathrm{min}$ ischemia in the rat 


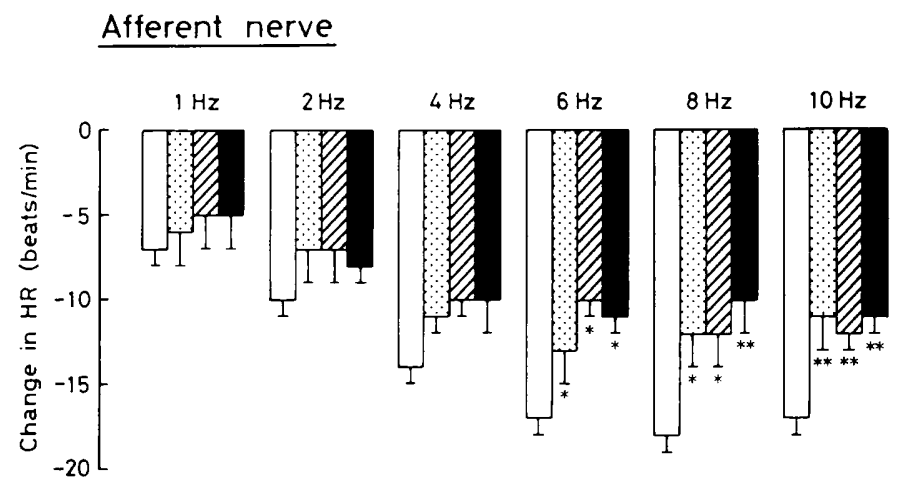

\section{Efferent nerve}

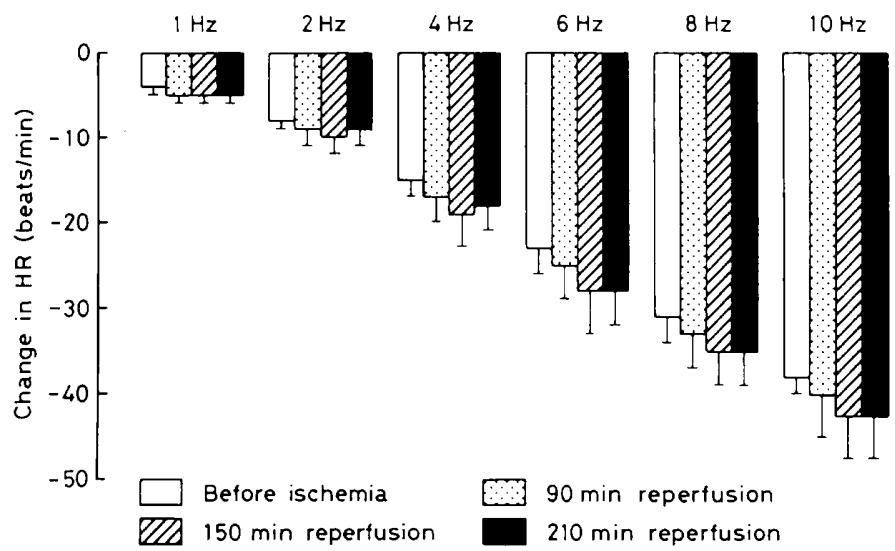

Fig. 6. Influence of 10-min cerebral ischemia on heart rate responses to the electrical stimulation of the left vagal afferent or efferent nerve. Each column consists of the results from 5 animals. ${ }^{*} P<0.05 .{ }^{*} P<$ 0.01 : significantly different from the value before ischemia.

forebrain ischemia model. Therefore, the present result cannot exclude the possibility that 5 -min ischemia might produce some impairment of the cortical EEG at a later period of reperfusion.

Transient cerebral ischemia is known to be followed by regionally heterogeneous circulatory disturbances such as the no-reflow phenomenon (13-15) and the post-ischemic hypoperfusion syndrome (15-20), which may disturb functional recovery of the brain. Therefore, another possible explanation would be that the degree of post-ischemic hypoperfusion in the baroreflex pathway might be greater than that in the cerebral cortex, resulting in the selective attenuation of the baroreflex activity. Furthermore, since incomplete ischemia is known to produce greater damage than complete ischemia (21). selective damage of the baroreflex pathway might be ascribed to the greater amount of residual blood flow in the baroreflex pathway during ischemia compared with that in the cerebral cortex. However, further investigation on the comparison of blood flow changes in the cerebral cortex and baroreflex pathway is necessary to know whether these two possible explanations are reasonable or not.

Finally, it can be also speculated that the regional difference in the functional activity during ischemia might be responsible for the selective attenuation of the baroreflex activity. In contrast with the rapid cessation of the cortical electrical activity by ischemia, 
the vasomotor center in the ventrolateral medulla as well as the cardioinhibitory center. i.e., nucleus ambiguus or dorsal motor nucleus, are excited by ischemia to produce the characteristic cerebral ischemic response $(22-28)$. Also in the present study, the ischemic procedure produced a marked increase in systemic blood pressure, which is one of the characteristics of the cerebral ischemic response. The degree of hypertension was significantly greater than that observed in the incomplete occlusion group and was quite similar to that observed by Schrader et al. (23) in pentobarbitalized dogs which received complete ischemic insult by supratentorial balloon expansion. Thus, it can be assumed that the extent of energy deficiency in the excited regions may be greater than other silent regions, and consequently, these excited regions are regarded to be more vulnerable to the ischemia. Since the regions which contribute to the cerebral ischemic response are also involved in the baroreflex pathway, it is likely that the cerebral ischemic response may be responsible for the selective attenuation of baroreflex activity following 5 -min ischemia. Recently, it was suggested that excessive accumulation of endogenously released glutamate or other excitatory amino acids may play a role in the pathogenesis of several neurologic diseases including ischemic neuronal injury (29). Furthermore, the receptors for glutamate have been shown to exist and to play a functional role in the central pathways of baroreflex such as the nucleus tractus solitali (30) or the ventrolateral medulla $(31,32)$. Thus, these findings make us speculate that attenuation of the baroreflex might be due to the excitotoxicity of excitatory amino acids excessively released and accumulated in the central pathway of the baroreflex during the ischemia.

In summary, the present study indicates that transient global cerebral ischemia of more than 5 min produces the significant attenuation of the baroreflex during the reperfusion period of 60 to $2.10 \mathrm{~min}$. The damaged site responsible for the attenuation of the baroreflex seems to be involved in the central pathway of the baroreflex mechamism, although the precise site of lesion or mechanism of attenuation is still under investigation.

Acknowledgments: Part of this study was supported by the Tanaka Memorial Medical Research Fund. The authors are grateful to Mr. Yuji Noguchi, Mr. Kazuyoshi Saito and Miss Midori Kawasaki for their skillful technical assistance.

\section{References}

1 Cropp, G.J. and Manning, G.W.: Electrocardiographic changes simulating myocardial ischemia and infarction associated with spontaneous intracranial hemorrhage. Circulation 22, 25-38 (1960)

2 Srivastava, S.C. and Robson, A.O.: Electrocardiographic abnormalities associated with subarachnoid haemorrhage. Lancet 2, 431-433 (1964)

3 Myers, M.G., Norris, J.W., Hachinski, V.C., Weingert, M.E. and Sole, M.J.: Cardiac sequelae of acute stroke. Stroke 13, 838-842 (1982)

4 Appenzeller, 0 . and Descarries, L: Circulatory reflexes in patients with cerebrovascular disease. N. Engl. J. Med. 271, 820-823 (1964)

5 Johnson, R.H., Smith, A.C., Spalding, J.M.K. and Wollner, L.: Effect of posture on bloodpressure in elderly patients. Lancet 1, 731-733 (1965)

6 Gross, M.: Circulatory reflexes in cerebral ischaemia involving different vascular territories. Clin. Sci. 38, 491-502 (1970)

7 Aukland, K., Bower, B.F. and Berliner, R.W.: Measurement of local blood flow with hydrogen gas. Circ. Res. 14, 164-187 (1964)

8 Lassen, N.A., Høedt-Rasmussen, K., Sørensen, S.C., Skinhøj, E., Cronquist, S., Bodforss, B., Eng, E. and Ingvar, D.H.: Regional cerebral blood flow in man determined by krypton ${ }^{85}$. Neurology 13, 719-727 (1963)

9 Hachinski, V.C., Smith, K.E., Silver, M.D., Gibson, C.J. and Ciriello, J.: Acute myocardial and plasma catecholamine changes in experimental stroke. Stroke 17, 387-390 (1986)

10 Ito, U., Spatz, M., Walker, J.T. and Klatzo, I.: Experimental cerebral ischemia in Mongolian gerbils. 1. Light microscopic observations. Acta Neuropathol (Berl.) 32, 209-223 (1975)

11 Suzuki, R., Yamaguchi, T., Li, C.-L. and Klatzo, I.: The effects of 5-minute ischemia in Mongolian gerbils: II. Changes of spontaneous neuronal activity in cerebral cortex and CA1 sector of hippocampus. Acta Neuropathol (Berl.) 60, 217-222 (1983)

12 Smith, M.-L., Auer, R.N. and Siesjö, B.K.: The density and distribution of ischemic brain injury in the rat following $2-10 \mathrm{~min}$ of forebrain 
ischemia. Acta Neuropathol (Berl.) 64, 319-332 (1984)

13 Ames, A., Wright, R.L., Kowada, M., Thurston, J.M. and Majno, G.: Cerebral ischemia. II. The no-reflow phenomenon. Am. J. Pathol. 52, 437453 (1968)

14 Fischer, E.G.: Impaired perfusion following cerebrovascular stasis. Arch. Neurol. 29, 361366 (1973)

15 Kågström, E., Smith, M.-L. and Siesjö, B.K.: Local cerebral blood flow in the recovery period following complete cerebral ischemia in the rat. J. Cereb. Blood Flow Metab. 3, 170-182 (1983)

16 Snyder, J.V., Nemoto, E.M., Carroll, R.G. and Safar, P.: Global ischemia in dogs: Intracranial pressures, brain blood flow and metabolism. Stroke 6, 21-27 (1975)

17 Ginsberg, M.D., Budd, W.W. and Welsh, F.A.: Diffuse cerebral ischemia in the cat: I. Local blood flow during severe ischemia and recirculation. Ann. Neurol. 3, 482-492 (1978)

18 Pulsinelli, W.A., Levy, D.E. and Duffy, T.E.: Regional cerebral blood flow and glucose metabolism following transient forebrain ischemia. Ann. Neurol. 11, 499-509 (1982)

19 Marcy, V.R. and Welsh, F.A.: Correlation between cerebral blood flow and ATP content following tourniquet-induced ischemia in cat brain. J. Cereb. Blood Flow Metab. 4, 362-367 (1984)

20 Todd, N.V., Picozzi, P., Crockard, H.A. and Russell, R.R.: Reperfusion after cerebral ischemia. Influence of duration of ischemia. Stroke 17, 460-466 (1986)

21 Siesjö, B.K.: Cerebral circulation and metabolism. J. Neurosurg. 60, 883-908 (1984)

22 Sagawa, K., Ross, J.M. and Guyton, A.C.: Quantitation of cerebral ischemic pressor response in dogs. Am. J. Physiol. 200, 11641168 (1961)

23 Schrader, H., Hall, C. and Zwetnow, N.N.: Effects of prolonged supratentorial mass expansion on regional blood flow and cardiovascular parameters during the Cushing response. Acta Neurol. Scand. 72, 283-294 (1985)
24 Dampney, R.A.L., Kumada, M. and Reis, D.J.: Central neural mechanisms of the cerebral ischemic response. Characterization, effect of brainstem and cranial nerve transections and simulation by electrical stimulation of restricted regions of medulla oblongata in rabbit. Circ. Res. 45, 48-62 (1979)

25 Kumada, M., Dampney, R.A.L. and Reis, D.J.: Profound hypotension and abolition cf the vasomotor component of the cerebral ischemic response produced by restricted lesions of medulla oblongata in rabbit. Relationship to the so-called tonic vasomotor center. Circ. Res. 45, 63-70 (1979)

26 Dampney, R.A.L. and Moon, E.A.: Role of ventrolateral medulla in vasomotor response to cerebral ischemia. Am. J. Physiol. 239, H349H358 (1980)

27 Guyenet, P.G. and Les Brown, D.: Unit activity in nucleus paragigantocellularis lateralis during cerebral ischemia in the rat. Brain Res. 364, 301-314 (1986)

28 Prabhakar. N.R., Mitra, J., Van De Graaff, W., Haxhiu, M.A. and Cherniack, N.S.: Effect of focal cooling of central chemosensitive areas on cerebral ischemic response. Am. J. Physiol. 251, R295-R302 (1986)

29 Rothman, S.M. and Olney, J.W.: Glutamate and the pathophysiology of hypoxic-ischemic brain damage. Ann. Neurol. 19, 105-111 (1986)

30 Talman, W.T., Perrone, M.H. and Reis, D.J.: Evidence for L-glutamate as the neurotransmitter of baroreceptor afferent nerve fibers. Science 209, 813-815 (1980)

31 Willette, R.N., Barcas, P.P., Krieger, A.J. and Sapru, H.N.: Vasopressor and depressor areas in the rat medulla. Identification by microinjection of L-glutamate. Neuropharmacology 22, 1071-1079 (1983)

32 Gordon, F.J.: Aortic baroreceptor reflexes are mediated by NMDA receptors in caudal ventrolateral medulla. Am. J. Physiol. 252, R628R633 (1987) 